ITP-UU-04/26, SPIN-04/14

\title{
Photon mass in inflation and nearly minimal magnetogenesis *
}

\author{
Tomislav Prokopec \\ Institute for Theoretical Physics 85 Spinoza Institute, Leuvenlaan 4, \\ 3584 CE Utrecht, The Netherlands; Email: T.Prokopec@phys.uu.nl
}

\begin{abstract}
We argue that the dynamics of photons canonically coupled to scalars in de Sitter inflation gets modified by a photon mass term. This might have lead to generation of potentially observable magnetic field correlated over cosmological scales today.
\end{abstract}

\section{INTRODUCTION}

One of the important unresolved puzzles of modern astrophysics and cosmology is the origin of large scale magnetic fields of the Universe. It is conceivable that galactic magnetic fields are explained by the dynamo mechanism, by which an initial small seed field at the time of galaxy formation was amplified to the microgauss strength observed today. Seed fields of magnitudes $B_{\text {seed }} \sim 10^{-20}$ Gauss and correlated over distances larger than about 100 parsecs could have been created by the Biermann battery mechanism induced by the large scale structure dynamics at the time of galaxy formation. On the other hand, it seems that no currently available astrophysical scenario can explain recent cluster fields [1] observed in several galaxy clusters.

Based on a study of scalar electrodynamics ( $\Phi \mathrm{ED}$ ) during inflation [2, 3, 4, 5, 6, 7, 8, 9] and subsequent radiation and matter epochs, in this talk I will argue that the observed large scale magnetic fields could have originated in cosmological inflation.

\footnotetext{
* Contribution to the Proceedings of Strong and Electroweak Matter 2004 (SEWM2004), Helsinki, Finland, June 16-19, 2004.
} 


\section{THE MODEL}

Cosmological inflation is an epoch of a rapid (accelerated) expansion of the Universe. For simplicity, here we model inflation by de Sitter space-time. In conformal coordinates it is characterized by the metric, $g_{\mu \nu}=a^{2} \eta_{\mu \nu}$, where $\eta_{\mu \nu}=\operatorname{diag}(-1,1,1,1)$ and the scale factor $a=-1 /(H \eta), H$ is the Hubble parameter and $\eta$ conformal time. In conformal coordinates the Lagrangian of scalar electrodynamics reduces to

$$
\mathbf{L}_{\Phi \mathrm{ED}}=-\frac{1}{4} \eta^{\mu \nu} \eta^{\rho \sigma} F_{\mu \rho} F_{\nu \sigma}-a^{2} \eta^{\mu \nu}\left(D_{\mu} \Phi\right)^{*}\left(D_{\nu} \Phi\right)-a^{4}\left(m_{\phi}^{2}+\xi \mathrm{R}\right) \Phi^{*} \Phi
$$

where $F_{\mu \rho}=\partial_{\mu} A_{\rho}-\partial_{\rho} A_{\mu}$ is the photon field strength, $D_{\mu}=\partial_{\mu}-i e A_{\mu}$ is the covariant derivative, $m_{\phi}$ the scalar field mass, $\mathrm{R}$ the curvature scalar, which in $D=4$ and de Sitter inflation reads $\mathrm{R}=12 H^{2}$, and $A_{\mu}$ is the gauge (photon) field. When $m_{\phi}^{2}+\xi \mathrm{R} \neq 0$, conformal invariance of $\Phi$ is broken during inflation, resulting in a growth of scalar fluctuations primarily on superhorizon scales. This is formally encoded by the Chernikov-Tagirov [1]] (Bunch-Davies) Feynman propagator, which in $D=4$ reads

$$
i G_{F}(y)=\frac{H^{2}}{4 \pi^{2}} \Gamma\left(\frac{3}{2}-\mu\right) \Gamma\left(\frac{3}{2}+\mu\right){ }_{2} F_{1}\left(\frac{3}{2}-\mu, \frac{3}{2}+\mu, 2 ; 1-\frac{y}{4}\right)
$$

where $\mu=\left[(3 / 2)^{2}-\left(m_{\phi}^{2}+\xi \mathrm{R}\right) / H^{2}\right]^{1 / 2}$, and $y=a(\eta) a\left(\eta^{\prime}\right) \Delta x^{2}$ is the de Sitter invariant length, $\Delta x^{2}=-\left(\left|\eta-\eta^{\prime}\right|-i \epsilon\right)^{2}+\left\|\vec{x}-\vec{x}^{\prime}\right\|^{2}$. When expanded in $s \equiv(3 / 2)-\mu \approx\left(m_{\phi}^{2} / 3 H^{2}\right)+4 \xi$ $(|s| \ll 1)$, the propagator simplifies to $[\underline{3}]$

$$
i G_{F}(y) \simeq \frac{H^{2}}{4 \pi^{2}}\left\{\frac{1}{y}-\frac{1}{2} \ln (y)+\frac{1}{2 s}-1+\ln (2)\right\} .
$$

Note that this propagator is still fully de Sitter invariant. In the limit when $m_{\phi} \rightarrow 0$ and $\xi \rightarrow 0$ however, the propagator diverges (2), and a natural choice is an $S O(3,1)$ invariant propagator [3, 12],

$$
\left.i G_{F}\right|_{m_{\phi}=0} \simeq \frac{H^{2}}{4 \pi^{2}}\left\{\frac{1}{y}-\frac{1}{2} \ln (y)+\frac{1}{2} \ln \left(a(\eta) a\left(\eta^{\prime}\right)\right)-\frac{1}{4}+\ln (2)\right\} .
$$

The de Sitter invariance $S O(4,1)$ is broken by the term $\propto \ln \left(a(\eta) a\left(\eta^{\prime}\right)\right)$. We shall use both propagators (3) and (4) to study how scalars influence the photon dynamics during inflation and its consequences today.

During inflation the photon dynamics in $\Phi E D$ (II) is governed by the modified Maxwell equation,

$$
\eta^{\mu \nu} \partial_{\mu} F_{\nu \rho}+\eta_{\rho \mu} \int d^{4} x^{\prime}\left[{ }^{\mu} \Pi_{\mathrm{ret}}^{\nu}\right]\left(x, x^{\prime}\right) A_{\nu}\left(x^{\prime}\right)=0
$$


where $\left[{ }^{\mu} \Pi_{\mathrm{rat}}^{\nu}\right]$ denotes the retarded vacuum polarization tensor of the theory. We have calculated [3, 5] the one-loop photon vacuum polarization tensor by making use of the Schwinger-Keldysh formalism, suitable for study of time dependent problems. Our polarization tensor is renormalized by the technique of dimensional regularization. Further, it is manifestly transverse, and hence gauge invariant. We have performed both calculations, for photons coupled to a massive light scalar (3) [3] , as well as to a massless minimally coupled scalar (44) [5]. In both cases the photon acquires a mass through the coupling to the infrared scalar modes. The result for the case of coupling to massive excitations can be neatly summarized by the effective Lagrangian [3]

$$
\mathbf{L}_{\Phi \mathrm{ED}}^{\mathrm{eff}}=\mathbf{L}_{\Phi \mathrm{ED}}-\frac{1}{2} a^{2} \eta^{\mu \nu} A_{\mu} A_{\nu}
$$

such that the photon obeys a Proca equation of motion, with the mass

$$
m_{\gamma}^{2} \simeq \frac{3 \alpha H^{4}}{\pi\left(m_{\phi}^{2}+\xi \mathrm{R}\right)}
$$

where $\alpha=e^{2} /(4 \pi)$ denotes the fine structure constant. This result is obtained in a generalized Loren $(\mathrm{t}) \mathrm{z}$ gauge, $\eta^{\mu \nu} \partial_{\mu}\left(a^{2} A_{\nu}\right)=0$. Both transverse and longitudinal vector excitations are endowed by the mass (77). This represents an alternative, gravity induced, mechanism for gauge field mass generation, in which the longitudinal photon degree of freedom is provided by the infrared modes of the (complex) scalar field. As a consequence of mass generation, scaling of the electric and magnetic fields during inflation changes from the vacuum scaling, $\vec{E}_{\mathrm{vac}}, \vec{B}_{\mathrm{vac}} \propto a^{-2}$ to $\vec{E} \propto a^{-\frac{3}{2}-\frac{1}{2}\left[1-4\left(m_{\gamma} / H\right)^{2}\right]^{1 / 2}}$ and $\vec{B} \propto a^{-\frac{5}{2}+\frac{1}{2}\left[1-4\left(m_{\gamma} / H\right)^{2}\right]^{1 / 2}}$. If the photon couples to a massless minimally coupled scalar, then the magnetic field remains massless, while the electric field acquires a time dependent mass, $m_{\gamma}^{2}(a)=\left(2 \alpha H^{2} / \pi\right)[\ln (a)-1 / 4]$, u] such that the fields scale differently, $\vec{E} \propto a^{-3 / 2}$ and $\vec{B} \sim \vec{B}_{\text {vac }} \propto a^{-2}$.

\section{NEARLY MINIMAL MAGNETOGENESIS}

We shall now argue that, provided the scalar field evolution remains de Sitter invariant (3), a certain choice of the couplings leads to magnetic field generation during inflation.

A general solution of the photon mode equation associated with the Proca effective theory (6) can be written in terms of the Hankel function,

$$
A_{\mu}=\frac{1}{2} \sqrt{-\pi \eta} H_{\nu}^{(1)}(-k \eta) \varepsilon_{\mu}^{(1)}, \quad \nu=\frac{1}{2}\left(1-4 m_{\gamma}^{2} / H^{2}\right)^{1 / 2}
$$


where $\varepsilon_{\mu}^{(1)}$ denotes the photon polarization vector. This needs to be matched to the plane wave solutions of the radiation era, $A_{\mu}^{(\mathrm{rad})}=(2 k)^{-1 / 2} \mathrm{e}^{\mp i k \eta}$. These modes describe correctly the photon dynamics in radiation era, provided conductivity is small and the photon mass decays nonadiabatically during radiation era. After performing the mode matching, we arrive at the following ensemble averaged magnetic field in radiation era,

$$
\left\langle\vec{B}^{2}(\vec{x}, \eta, \ell)\right\rangle=\int \frac{d k}{k} \mathbf{P}_{B}(k, \eta, \ell)|w(k, \ell)|^{2},
$$

where $w$ denotes a window function, e.g. $\quad w=\exp \left(-k^{2} \ell^{2} / 2\right) \equiv \int d^{3} x W(\vec{x}) \exp (i \vec{k} \cdot \vec{x})$, $W=\left(2 \pi \ell^{2}\right)^{-3 / 2} \exp \left(-\|\vec{x}\| /\left(2 \ell^{2}\right)\right)$, and $\mathbf{P}_{B}(k, \eta, \ell)$ is the field spectrum (defined in analogous manner as the spectrum of cosmological perturbations),

$$
\mathbf{P}_{B}=\frac{1}{a^{4}} \frac{\Gamma^{2}(\nu)}{8 \pi^{3}}\left(\nu-\frac{1}{2}\right)^{2}(2 H)^{2 \nu+1} k^{3-2 \nu} \sin ^{2}((k / H)(a-1)) .
$$

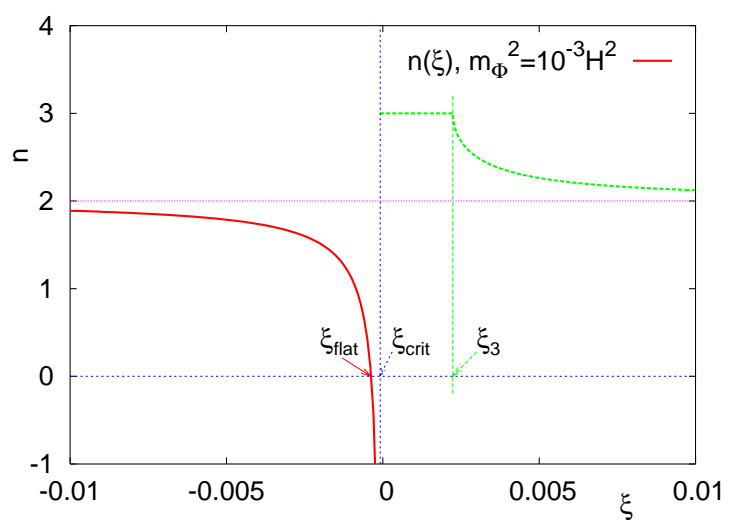

FIG. 1: Spectral index $n$ as a function of the scalar field coupling to curvature $\xi$ and the scalar $\operatorname{mass} m_{\phi}^{2}$.

This spectrum is characterized by the spectral index $n=3-2 \nu$ on subhorizon scales, and by $n=5-2 \nu$ on superhorizon scales. Figure 1 shows $n$ on subhorizon scales as a function of $\xi$ and $m_{\phi}^{2}$. Note that for $\xi_{\text {flat }}=-(\alpha / 8 \pi)-m_{\phi}^{2} / 12 H^{2}$, one gets a flat spectrum, $n=0$. In this case the field strength is typically $B \sim 10^{-10}$ Gauss on all scales, such that it is potentially observable by the next generation of CMBR measurements. The region of couplings $\xi \in$ $\left(\xi_{\text {flat }}, \xi_{\text {crit }}\right) \quad\left(\xi_{\text {crit }}=-m_{\phi}^{2} / 12 H^{2}\right)$ leads to a growth in magnetic energy during inflation, such that its backreaction on the background space-time may become important. This may change the course of the Universe's evolution, and thus $n<0$ cannot be trusted. The values corresponding to $\xi>\xi_{3}=(\alpha / \pi)-m_{\phi}^{2} / 12 H^{2}$ have already been proposed earlier [7, 8, 9] as a model for magnetic field generation, powerful enough to seed the galactic dynamo. 
Finally, we recall that radiation era may be endowed by a large conductivity. In this case the photon dynamics is governed by the suitably modified [2] Bödeker-Langevin theory [10],

$$
\left(a \sigma \partial_{t}+\vec{k}^{2}\right) A^{T}=a^{3} \zeta^{T},\left\langle\zeta^{T}(\vec{k}, \eta) \zeta^{T^{\prime}}\left(\vec{k}^{\prime}, \eta\right)\right\rangle=\frac{2 \sigma T}{a^{4}} \delta^{T T^{\prime}}(2 \pi)^{3} \delta\left(\eta-\eta^{\prime}\right) \delta\left(\vec{k}-\vec{k}^{\prime}\right)
$$

and the spectrum (10) is modified to

$$
\mathbf{P}_{B}=\frac{1}{a^{4}} \frac{\Gamma^{2}(\nu)}{2 \pi^{3}}(2 H)^{2 \nu-1} k^{5-2 \nu} \exp \left(-\frac{2 k^{2}}{H \sigma}(1-1 / a)\right)+\mathbf{P}_{B}^{\text {th }} .
$$

This suggests the following definition of the conductivity momentum, $k_{\sigma}=\sigma H(t) / 2$, above which the plasma conductivity destroys any primordial spectrum. Neglecting the thermal contribution, $\mathbf{P}_{B}^{\text {th }} \propto k^{3}\left(k \gg k_{\sigma}\right)$, it is worth noting that the spectrum (12) gives a magnetic field that is smaller than (10) on all scales. The spectrum (10) may be understood as an upper bound and the spectrum (12) as a lower bound on the magnetic field spectrum obtained by a more realistic treatment of the photon dynamics during radiation and matter eras.

[1] C. Vogt and T. A. Ensslin, arXiv:astro-ph/0309441.

[2] T. Prokopec and E. Puchwein, Phys. Rev. D 70 (2004) 043004 arXiv:astro-ph/0403335.

[3] T. Prokopec and E. Puchwein, JCAP 0404 (2004) 007 arXiv:astro-ph/0312274.

[4] T. Prokopec and R. P. Woodard, Annals Phys. 312 (2004) 1 arXiv:gr-qc/0310056.

[5] T. Prokopec, O. Törnkvist and R. P. Woodard, Annals Phys. 303 (2003) 251-274 arXiv:gr-qc/0205130.

[6] T. Prokopec, O. Törnkvist and R. P. Woodard, Phys. Rev. Lett. 89, 101301 (2002) arXiv:astro-ph/0205331.

[7] T. Prokopec and R. P. Woodard, Am. J. Phys. 72 (2004) 60 arXiv:astro-ph/0303358.

[8] K. Dimopoulos, T. Prokopec, O. Törnkvist and A.-Ch. Davis, Phys. Rev. D 65 (2002) 063505 arXiv:astro-ph/0108093.

[9] A.-Ch. Davis, K. Dimopoulos, T. Prokopec and O. Törnkvist, Phys. Lett. B 501 (2001) 165 arXiv:astro-ph/0007214.

[10] D. Bödeker, Nucl. Phys. B 559 (1999) 502 arXiv:hep-ph/9905239.

[11] N. A. Chernikov and E. A. Tagirov, Annales Poincare Phys. Theor. A 9 (1968) 109.

[12] B. Allen and A. Folacci, Phys. Rev. D 35 (1987) 3771. 